\title{
VARIOUS LEVELS OF MODELS FOR AEROSOLS
}

\author{
Pierre-Emmanuel JABIN, \\ email: jabin@dma.ens.fr \\ Département de Mathématiques et Applications, École Normale Supérieure \\ 45 rue d'Ulm, 75230 Paris Cedex 05, France
}

(Leave 1 inch blank space for publisher.)

\begin{abstract}
Two limit behaviours of a simple model of aerosol are considered. The only force acting on aerosol particles is a friction due to the flow of gas. It is first proved that in the limit of an infinite friction coefficient, the particles are simply advected by the gas. Then we consider very dilute sprays of aerosol, i.e. with distribution functions which are monokinetic (Dirac mass in velocity). This approach leads to a macroscopic system with a free-boundary problem.
\end{abstract}

\section{Introduction}

The most general model is introduced in the first subsection under the form of a kinetic equation describing the transport of aerosols and their interaction with the surrounding gas. After that, we detail the two limits of this model which are studied.

\subsection{The general model}

Aerosol particles consist in some small droplets of a given product (typically a liquid or a gas), advected by a gas. The gas has no specific property in which we are interested. We only care about its dynamics because it acts on the aerosol particles of which we want to know the distribution.

Here we are interested in dilute sprays for relatively small droplets (of the order of $5-50 \mu \mathrm{m})$. This simplifies a lot the modelling because we may assume that there only exists an interaction of the advecting gas on the aerosol particles (no influence of the particles on the gas and no interaction between the particles). The only force acting on the aerosol particles is therefore a friction with the gas (the particles are not small enough for brownian motion to appear). The dynamics of the gas is fully represented by a given velocity field $u$, which is usually a solution of Navier-Stokes 
equation. It is also possible to consider gravity, but this does not change the form of the equation: It only means we should add a given constant to $u$. However we completely neglect all other phenomena like possible collisions between aerosol particles or coagulation-fragmentation process which could very weel happen even in a dilute spray.

The aerosol spray is modelled by the distribution function $f$ of the particles in the phase space $\Omega \times \mathbb{R}^{d}, \Omega$ being a given, regular subdomain of $\mathbb{R}^{d}$, with $d=2,3$ for pratictal applications.

Throughout this paper, we assume that this function $f$ satisfies the equation

$$
\begin{aligned}
& \frac{\partial f}{\partial t}+v \cdot \nabla_{x} f+\lambda \operatorname{div}_{v}((u-v) f)=0, \quad(t, x, v) \in \mathbb{R}_{+} \times \Omega \times \mathbb{R}^{d}, \\
& f(t=0, x, v)=f^{0}(x, v),
\end{aligned}
$$

where $\lambda$ is the friction coefficient and it depends on the viscosity of the gas, the size of the droplets and the ratio of the densities of the gas and of the aerosol particles. A typical application of this model is aerosols in the respiratory tract. The main question is the deposition of aerosol particles (see the paper by D.B. Taulbee and C.P. Yu [18] for more details). The aerosol is absorbed by the tract, but the location where it is absorbed is very important for the product to have the intended effects. Consequently, we consider domains $\Omega$ which are regular and bounded subdomain of $\mathbb{R}^{d}$. However, notice that all the results given here are also true if $\Omega$ consists in $\mathbb{R}^{d}$. In the case where $\Omega$ is not the whole space $\mathbb{R}^{d}$, we impose the boundary condition $(n(x)$ being the normal to $\partial \Omega$ at $x)$

$$
f(t, x, v)=0 \quad \text { if } v \cdot n(x) \leq 0, \quad \forall(t, x, v) \in[0, T] \times \partial \Omega \times \mathbb{R}^{d} .
$$

This condition means that any aerosol particle touching the boundary is immediately absorbed.

The initial data $f^{0}$ satisfies these natural estimates

$$
\begin{aligned}
& f^{0} \geq 0, \quad f^{0} \in L^{1} \cap L^{\infty}\left(\Omega \times \mathbb{R}^{d}\right), \\
& E^{0}=\int_{\Omega \times \mathbb{R}^{d}}|v|^{2} f^{0}(x, v) d x d v<+\infty .
\end{aligned}
$$

As for the velocity field $u$ of the gas, we assume that it has the following regularity

$$
u \in L^{2}\left([0, T], C^{0}(\Omega)\right)
$$

Notice that, if $u$ is a Leray solution of Navier-Stokes in dimension three, it only belongs to $L^{1}\left([0, T], C^{0}(\Omega)\right)$ a priori (see [14] for more details).

We only consider solutions $f$, limit of classical solutions to (1.1) and satisfying

$$
\left(1+|v|^{2}\right) f \in L^{\infty}\left([0, T], L^{1}\left(\Omega \times \mathbb{R}^{d}\right)\right), \quad f \in L^{\infty}\left([0, T], L^{\infty}\left(\Omega \times \mathbb{R}^{d}\right)\right) .
$$

With Estimates (1.3) and (1.4), it has already been proved that such solutions always exist (see [8]). Of course the boundary condition (1.2) is then interpreted in 
a weak sense. More precisely, it means that for any $R>0$ and any $\phi \in C^{1}([0, T] \times$ $\Omega \times B(0, R))$ with $\phi(t, x, v) \geq 0$, for all $x \in \partial \Omega$ such that $v \cdot n(x) \geq 0$, we have

$$
\int\left(-\frac{\partial \phi}{\partial t}-v \cdot \nabla_{x} \phi-\lambda(u-v) \operatorname{div}_{v} \phi\right) f d x d v d t \leq \int \phi(0, x, v) f^{0} d x d v .
$$

Eq. (1.1) is linear and relatively simple, it can be compared with other kinetic equations modeling particles in a fluid (see [11] and [13]). It is nevertheless posed in the phase space and it is quite costly to solve it numerically although a numerical approach is necessary to compute the deposition of aerosol. As a consequence, this paper aims at rigorously derive macroscopic systems, i.e. systems of equations in the physical space only wich are less costly from a numerical point of view. The main macroscopic quantities of interest are the density and current which are defined as

$$
\begin{aligned}
& \rho(t, x)=\int_{\mathbb{R}^{d}} f(t, x, v) d x d v, \\
& j(t, x)=\int_{\mathbb{R}^{d}} v f(t, x, v) d x d v .
\end{aligned}
$$

These quantities are connected by the continuity equation, obtained from (1.1) by integrating in velocity

$$
\frac{\partial \rho}{\partial t}+\operatorname{div}_{x} j=0
$$

We need one other relation on $j$. This is possible in two limit cases studied here, where the distribution function exhibits a Dirac mass in velocity. In the first case, we perform the limit of infinite friction coefficients for which the velocity of the aerosol is given by the velocity field $u$ of the gas. In the second case, we consider aerosol sprays so dispersed that the distribution function can be expressed on the form $\rho \delta(v-V(t, x))$. The equations satisfied by $\rho$ and $V$ are local with a free boundary.

\subsection{The limit of a large friction coefficient}

A first natural remark to do concerning Eq. (1.1) is that the friction coefficient $\lambda$ is very large since we consider small particles (if the particles are spheres of diameter $r, \lambda$ behaves as $r^{-1}$ ). This raises the question of what is happening as the coefficient converges toward infinity.

We thus consider the following system

$$
\begin{aligned}
& \frac{\partial f^{\varepsilon}}{\partial t}+v \cdot \nabla_{x} f^{\varepsilon}+\frac{1}{\varepsilon} \operatorname{div}_{v}\left((u-v) f^{\varepsilon}\right)=0 \\
& f^{\varepsilon}(t=0, x, v)=f^{0}(x, v) .
\end{aligned}
$$

Notice that the initial data $f^{0}$ does not depend on $\varepsilon$ : It is not prepared. At the limit $\varepsilon \rightarrow 0$, all the particles are advected with the velocity $u$ as it is proved by the theorem 
Theorem 1.1 Assume (1.3) and (1.4). Then for any $T>0$ and any sequence of solutions $f^{\varepsilon}$ to (1.9), satisfying (1.5), there exists $\rho \in L^{\infty}\left([0, T], M^{1}(\Omega)\right)$ such that

(i) $f^{\varepsilon} \longrightarrow \rho(t, x) \delta(v-u(t, x)) \quad$ in $w-M^{1}\left([0, T] \times \Omega \times \mathbb{R}^{d}\right)$,

(ii) $\rho^{\varepsilon} \longrightarrow \rho(t, x) \quad$ in $w-L^{\infty}\left([0, T], M^{1}(\Omega)\right)$ with

$$
\left\{\begin{array}{l}
\frac{\partial \rho}{\partial t}+\operatorname{div}_{x}(\rho u)=0 \\
\rho(t=0, x)=\int f^{0}(x, v) d v
\end{array}\right.
$$

\section{Remarks.}

1) The product $\rho \delta(v-u)$ is well defined as a measure on $[0, T] \times \Omega \times \mathbb{R}^{d}$ because of (1.4). In fact $u \in L^{1}\left([0, T], C^{0}\left(\mathbb{R}^{d}\right)\right)$ would be enough for that, but we need the $L^{2}$ in time regularity to prove the convergence.

2) If we also assume $u$ to be divergence free, then Eq. (1.10) propagates all $L^{p}$ norms of $\rho$. Consequently, since $\rho^{0}$ belongs to $L^{(d+2) / d}$, we know that $\rho$ belongs to $L^{\infty}\left([0, T], L^{(d+2) / d}\right)$.

This result is similar to the one obtained in [12] for a non linear equation modelling spherical particles using a fluid (at rest at infinity) as a mean of interaction between themselves. It is not known if the regularity assumption (1.4) on $u$ can be relaxed a bit. In one dimension and for a non linear equation, T. Goudon was able to prove rigorously a limit of the same kind but with a singular interaction kernel (see [7]). However in a linear case as we consider here, it seems necessary that $u$ be at least continuous in space to define the product $\rho u$.

Limits of kinetic equations with a strong force term have also been studied in other situations: by Frénod and Sonnendrücker in [5] (fixed magnetic force) and by Golse and Saint-Raymond in [6] (the gyrokinetic limit). The quasi-neutral limit of the Vlasov-Poisson system also leads to distribution functions which concentrate on a Dirac mass in velocity (see the papers by Y. Brenier [2] and E. Grenier [9] and [10]). In a sense, Theorem 1.1 illustrates the inconvenient of this approach of "infinite" friction coefficient. Indeed the flow of aerosol particles simply follows the flow $u$ of gas. However this flow $u$ precisely vanishes near the boundary $\partial \Omega$, since the gas typically satisfies Navier-Stokes equations (and in any case, it cannot leave the domain $\Omega$ except for gravity).

But this has for consequence that there is no deposition of aerosol particles in this limit. This is not in contradiction with intuition or experiment but it tells us that we should consider large but finite friction coefficients.

\subsection{Macroscopic equations for very dilute aerosols}

In general the aerosol particles are very dispersed in the gas, at least initially. The kinetic description is not very appropriate because, at one given point in space, 


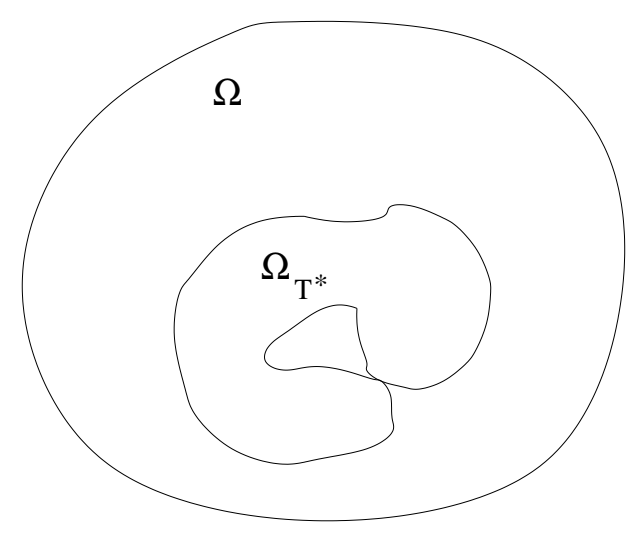

Figure 1: Definition of the time $T^{*}$.

there is only one particle whose velocity is uniquely determined by the position. Consequently we want to consider monokinetic distribution $f^{0}$. More precisely, we assume that

$$
f^{0}(x, v)=\rho^{0}(x) \delta\left(v-V^{0}(x)\right),
$$

whereas the boundary condition (1.2) is rewritten

$$
\rho^{0}(x) V^{0}(x) \cdot n(x) \geq 0 \quad \forall x \in \partial \Omega .
$$

We want to know if this precise monokinetic form $f=\rho(t, x) \delta(v-V(t, x))$ of the ditribution is maintained in time and, if it is so, which system of equations the couple $\rho, V$ satisfies. Notice that the field $V$ is defined only where $\rho$ does not vanish. Therefore, it is convenient to introduce here the set $\Omega_{t}$

$$
\Omega_{t}=\{x \in \Omega, \rho(t, x)>0\},
$$

and the first folding time $T^{*}$, i.e. when two different points of $\partial \Omega_{t}$ coincide (see figure 1). The time $T^{*}$ is precisely defined as the time up to which every connected part of the boundary $\partial \Omega_{t}$ remains Lipschitz and no separate parts of $\partial \Omega_{t}$ collide, i.e. no parts separate at some time $s<t$ become reunited at time $t$.

We may formally derive a system of equations for $\rho$ and $V$ by replacing $f$ by its particular form in Eq. (1.1). We obtain

$$
\left\{\begin{array}{l}
\frac{\partial \rho}{\partial t}+\operatorname{div}_{x}(\rho V)=0, \quad \forall x \in \Omega, \\
\frac{\partial V}{\partial t}+V \cdot \nabla_{x} V+\lambda(V-u)=0, \quad \forall x \in \Omega_{t}, \\
\rho(t, x) V(t, x) \cdot n(x) \geq 0, \quad \forall x \in \partial \Omega, \\
\rho(0, x)=\rho^{0}(x), \quad V(0, x)=V^{0}(x), \quad \forall x \in \Omega,
\end{array}\right.
$$


This system is a free boundary problem of a simple form, because the equations are all local: The derivate $\partial_{t} V$ at one point does not depend on the form of $\Omega_{t}$ and as for this domain, its boundary is simply moved with the velocity $V$.

Eq. (1.1) for monokinetic distributions may be interpreted as a kinetic formulation of System (1.14). Kinetic formulations of macroscopic systems have already been developed in other settings, particularly for Euler equation (see the papers by Y. Brenier [3], C. Dietz and V. Sandor [4] and P. Smereka [17])or pressureless gas dynamics, see F. Bouchut [1].

The distribution $f$ will remain monokinetic if two particles, initially at two different positions, never occupy the same position again. We face a problem which is in some sense close to geometric optic: The rays are well defined up to the point where they cross (see the paper by O. Runborg [16]). It is quite clear that in general the characteristics will not cross only if the velocity field $V(t, x)$ is regular enough (Lipschitz in fact). Since we still work with large friction coefficients (and thus with velocity fields "close" to $u$ ), we assume here that

$$
\begin{aligned}
& \left\|V^{0}\right\|_{W^{1, \infty}\left(\Omega_{0}\right)}<\infty, \\
& \|u\|_{L^{\infty}\left([0, T], W^{1, \infty}(\Omega)\right)}<\infty .
\end{aligned}
$$

Eq. (1.1) is linear and it makes sense, in the sense of distributions, even for solutions $f$ which are only measures. So there is one unique, global in time solution $f \in$ $L^{\infty}\left([0, T], M^{1}\left(\Omega \times \mathbb{R}^{d}\right)\right)$ to Eq. (1.1), satisfying the initial condition (1.11) and the boundary condition (1.2). If $f$ is monokinetic on the time interval $\left[0, T^{*}\right]$, the typical regularity we expect and need from the functions $\rho$ and $V$ is

$$
\begin{aligned}
& \rho \in L^{\infty}\left(\left[0, T^{*}\right], L^{1}(\Omega)\right), \\
& \forall t \leq T^{*}, \quad \Omega_{t} \subset \Omega \text { is an open domain with Lipschitz boundary, } \\
& V \in L^{\infty}\left(\left[0, T^{*}\right], W^{1, \infty}\left(\Omega_{t}\right)\right) .
\end{aligned}
$$

It is in fact easy to see that the solution $f$ remains monokinetic if there exists a couple of regular enough xolutions to (1.14)

Theorem 1.2 Given $\rho, V$ and $\Omega_{t}$ satisfying (1.17), it is equivalent to say that they solve System (1.14), and that the solution $f$ to (1.1) with (1.2) and (1.11) is exactly

$$
f(t, x, v)=\rho(t, x) \delta(v-V(t, x)) .
$$

\section{Remarks.}

1) Notice that Eq. (1.1) is linear and posed in the phase space whereas System (1.14) is non linear but posed in the real space.

2) The first equation of (1.14) is posed in the sense of distributions. So just as for Eq. (1.1), it is to be understood with the boundary condition as the following: for any $\phi \in C^{1}([0, T], \Omega)$ with $\phi(T,)=$.0 and $\phi(t, x) \geq 0, \forall x \in \partial \Omega$,

$$
\int_{0}^{T} \int_{\Omega} \rho(t, x)\left(-\frac{\partial \phi}{\partial t}-V \cdot \nabla_{x} \phi\right) d x d t \leq \int_{\Omega} \phi(0, x) \rho^{0}(x) d x .
$$


3) Formally, System (1.14) could also be written under the weaker form

$$
\left\{\begin{array}{l}
\frac{\partial \rho}{\partial t}+\operatorname{div}_{x}(\rho V)=0, \quad \forall x \in \Omega \\
\frac{\partial \rho V}{\partial t}+\operatorname{div}_{x}(\rho V \otimes V)+\lambda \rho(V-u)=0, \quad \forall x \in \Omega \\
\rho(t, x) V(t, x) \cdot n(x) \geq 0, \quad \forall x \in \partial \Omega .
\end{array}\right.
$$

This system requires less regularity on $V$. However, if $\rho$ and $V$ are only solutions to (1.19), a priori nothing ensures that $\rho \delta(v-V)$ is a solution to (1.1). This corresponds to the intuition that $f$ remains monokinetic and that we can prove uniqueness for (1.19), only if $V$ is Lipschitz. And of course if $V \in W^{1, \infty}$, then Systems (1.14) and (1.19) are equivalent.

It remains to prove existence for System (1.14) with the theorem

Theorem 1.3 Assume that $\rho^{0} \in L^{1}(\Omega)$ and

$$
\begin{aligned}
& \left\{x \in \Omega, \rho^{0}(x)>0\right\} \subset \Omega \text { is open with Lipschitz boundary, } \\
& \left\|V^{0}\right\|_{W^{1, \infty}\left(\Omega_{0}\right)} \leq \frac{\lambda}{2 d}, \quad \rho^{0} V^{0} \cdot n \geq 0 \text { on } \partial \Omega \\
& \|u\|_{L^{\infty}\left([0, T], W^{1, \infty}(\Omega)\right)} \leq \frac{\lambda}{4 d}
\end{aligned}
$$

Then there exists unique $\rho, V$ and $\Omega_{t}$ defined on a maximal time interval $\left[0, T^{*}\right)$, satisfying (1.17) and solutions to (1.14). The time $T^{*}$ is exactly the first folding time and is bounded from below by a constant independent on $\lambda$.

\section{Remarks.}

1) In fact, we use Eq. (1.1) to prove a short time existence result for System (1.14) and then, a priori estimates to extend it to the time $T^{*}$. From this point of view, Theorem 1.3 should be seen as an existence result for small initial data. Of course, this data has to be small in comparison with the friction coefficient which, in practice, is large so it is not a very demanding condition.

2) For a bounded domain $\Omega$, it is not a priori possible to pose the equation

$$
\frac{\partial V}{\partial t}+V \cdot \nabla V+\lambda(V-u)=0
$$

in the whole domain $\Omega$, even if initially $\rho^{0}>0$ everywhere. Indeed the boundary condition $V \cdot n \geq 0$ would not prevent the particles from leaving the domain (which corresponds to what we want) and in particular vacuum could appear (regions with no aerosol particles).

3) We do not impose that $\omega_{0}$ be connected. Indeed because we work on a bounded domain $\Omega$, this condition may be lost with the evolution in time (see figure 2 for a possible example). 


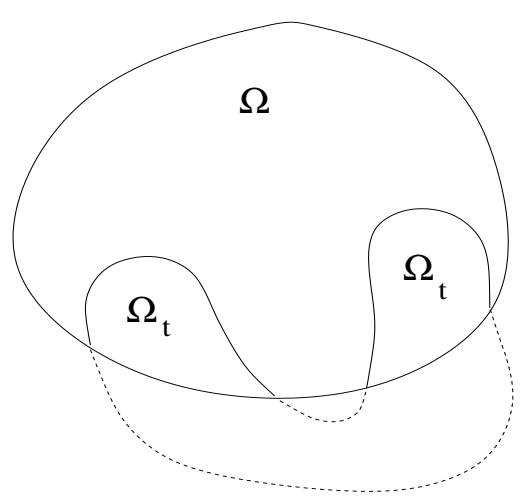

Figure 2: Example of loss of connectivity: $\Omega_{t}$ was simply translated from $\Omega_{0}$ and the dots represent the parts of the translation of $\partial \Omega_{0}$ "cut" by $\Omega$.

\subsection{Very dilute aerosol and large friction coefficients}

We deal here with the combination of the two previous approaches: We investigate the limit of infinite coefficients $\lambda$ in (1.14). First of all, since the existence time for this system is bounded from below independently of $\lambda$, we may work on a fixed time interval $[0, T]$ where System (1.14) has a unique solution. We thus consider the sequence of solutions on $[0, T]$ satisfying

$$
\left\{\begin{array}{l}
\frac{\partial \rho_{\varepsilon}}{\partial t}+\operatorname{div}_{x}\left(\rho_{\varepsilon} V_{\varepsilon}\right)=0, \quad \forall x \in \Omega \\
\frac{\partial V}{\partial t}+V \cdot \nabla_{x} V_{\varepsilon}+\frac{1}{\varepsilon}\left(V_{\varepsilon}-u\right)=0, \quad \forall x \in \Omega_{t}^{\varepsilon} \\
\rho_{\varepsilon}(t, x) V_{\varepsilon}(t, x) \cdot n(x) \geq 0, \quad \forall x \in \partial \Omega \\
\rho_{\varepsilon}(0, x)=\rho^{0}(x) \in L^{1}\left(\mathbb{R}^{d}\right), \quad V_{\varepsilon}(0, x)=V^{0}(x) \in W^{1, \infty}\left(\Omega_{0}\right), \quad \forall x \in \Omega
\end{array}\right.
$$

where as before

$$
\Omega_{t}^{\varepsilon}=\left\{x \in \Omega, \rho_{\varepsilon}(t, x)>0\right\} .
$$

Taking the formal limit, we obtain the equation

$$
\begin{cases}\frac{\partial \rho}{\partial t}+\operatorname{div}_{x}(\rho u)=0, & \forall x \in \Omega, \forall t \geq 0, \\ \rho(t=0, x)=\rho^{0}(x), & \forall x \in \Omega .\end{cases}
$$

This limit can be made rigorous in the following sense 
Theorem $1.4 A s \varepsilon \rightarrow 0$, we have for the solutions $\left(\rho_{\varepsilon}, V_{\varepsilon}, \Omega_{t}^{\varepsilon}\right)$ to (1.21)

(i) $\rho_{\varepsilon} \longrightarrow \rho$ solution to (1.23), in $w-M^{1}(\Omega)$,

(ii) $\mathbf{I}_{\Omega_{t}^{\varepsilon}}\left(V_{\varepsilon}-u\right) \longrightarrow 0$ in $\mathrm{L}^{p}(\Omega), \forall p<\infty$,

(iii) $\phi_{\varepsilon}\left(\frac{V_{\varepsilon}-u}{\varepsilon}-\partial_{t} u+u \cdot \nabla_{x} u\right) \longrightarrow 0$ in $W^{-1,1}([0, T] \times \Omega)$, for any $\phi_{\varepsilon}$ bounded uniformly in $\varepsilon$ in $W^{1, \infty}([0, T] \times \Omega)$ with supp $\phi_{\varepsilon}(t,.) \subset \Omega_{t}^{\varepsilon}$.

\section{Remark.}

The point ( $i i i)$ is a first order correction. If $u$ satisfies Euler equation, then $V_{\varepsilon}=$ $u+\varepsilon \nabla p$ where $p$ is the pressure field: it takes into account the inertia of the aerosol particles and so the forces acting inside the fluid appear.

\section{Proof of Theorem 1.1}

We first show some uniform, easy $L^{1}$ estimates for the sequence $f^{\varepsilon}$. After that we explain why some modified kinetic energy converges toward zero, which will give the result.

\section{$2.1 \quad$ Uniform estimates}

We first recall here that (because of the boundary condition (1.2) if $\Omega \neq \mathbb{R}^{d}$ )

$$
\left\|f^{\varepsilon}\right\|_{L^{\infty}\left([0, T], L^{1}\left(\Omega \times \mathbb{R}^{d}\right)\right)}=\left\|\rho^{\varepsilon}\right\|_{L^{\infty}\left([0, T], L^{1}(\Omega)\right)} \leq\left\|f^{0}\right\|_{L^{1}\left(\Omega \times \mathbb{R}^{d}\right)} .
$$

This implies that, up to the extraction of a subsequence, there exists $f \in L^{\infty}([0, T]$, $\left.M^{1}\left(\Omega \times \mathbb{R}^{d}\right)\right)$ and $\rho \in L^{\infty}\left([0, T], M^{1}(\Omega)\right)$ such that $f$ and $\rho$ are the weak limits of $f^{\varepsilon}$ and $\rho^{\varepsilon}$.

We can moreover prove that the kinetic energy $E^{\varepsilon}(t)=\int|v|^{2} f^{\varepsilon} d x d v$ is uniformly bounded in $\varepsilon$. Indeed we have from the equation (1.9), formally at least

$$
\frac{d}{d t} \int_{\Omega \times \mathbb{R}^{d}}|v|^{2} f^{\varepsilon} d x d v=-\frac{2}{\varepsilon} \int|v|^{2} f d x d v+\frac{2}{\varepsilon} \int u \cdot j d x-\int_{\partial \Omega \times \mathbb{R}^{d}}|v|^{2} v \cdot n(x) f^{\varepsilon} .
$$

And of course

$$
\begin{aligned}
\int u \cdot j d x & =\int u \cdot v f d x d v \leq\left(\int|u|^{2} \rho d x\right)^{1 / 2}\left(\int|v|^{2} f d x d v\right)^{1 / 2} \\
& \leq\|u(t, .)\|_{L^{\infty}(\Omega)}\left\|f^{0}\right\|_{L^{1}}^{1 / 2} \sqrt{E^{\varepsilon}(t)}
\end{aligned}
$$

So as a consequence and because of the boundary condition (1.2)

$$
E^{\varepsilon}(t) \leq E^{0}-\frac{2}{\varepsilon} \int_{0}^{t} E^{\varepsilon}(s) d s+\frac{C}{\varepsilon}\|u\|_{L^{2}\left([0, T], L^{\infty}\right)}\left(\int_{0}^{t} E^{\varepsilon}(s) d s\right)^{1 / 2} .
$$


A slighty modified Gronwall lemma then shows that

$$
\int_{0}^{T} E^{\varepsilon}(t) d t \leq C
$$

This computation is, a priori, only formal, i.e. valid only for regular solutions $f^{\varepsilon}$. However because of Assumption (1.5), the conclusion (2.2) is true for any solution, within the hypothesis of Theorem 1.1.

The energy bound implies that the relation (1.6) is also true at the limit

$$
\rho(t, x)=\int_{\mathbb{R}^{d}} f(t, x, v) d x d v,
$$

and also that the current $j^{\varepsilon}$ is uniformly bounded in $L^{\infty}\left([0, T], L^{1}(\Omega)\right)$ and so

$$
j^{\varepsilon} \longrightarrow j(t, x)=\int_{\mathbb{R}^{d}} v f(t, x, v) d v, \quad \text { in } w-L^{\infty}\left([0, T], M^{1}(\Omega)\right) .
$$

\subsection{Convergence of a modified kinetic energy}

We are interested in the quantity

$$
\tilde{E}^{\varepsilon}(t)=\int_{\Omega \times \mathbb{R}^{d}}|v-u(t, x)|^{2} f^{\varepsilon}(t, x, v) d x d v .
$$

We are going to prove that $\tilde{E}^{\varepsilon}$ converges toward 0 in $L^{1}([0, T])$. To do so, we approximate $u$ in $L^{2}\left([0, T], C^{0}(\Omega)\right)$ by a sequence $u_{n}$ of $C^{\infty}$ functions and we consider the modified energies $\tilde{E}_{n}^{\varepsilon}$ (defined with $u_{n}$ instead of $u$ ). Thanks to the bound (2.2), the sequence $\tilde{E}_{n}^{\varepsilon}$ converges toward $\tilde{E}^{\varepsilon}$ in $L^{1}([0, T])$, uniformly in $\varepsilon$. Now for a fixed $n$, we use Eq. (1.9) to estimate

$$
\begin{aligned}
\frac{d}{d t} \tilde{E}_{n}^{\varepsilon} & =2 \int \partial_{t} u_{n} \cdot\left(u_{n}-v\right) f^{\varepsilon}+2 \int\left(v \cdot \nabla_{x}\right) u_{n} \cdot\left(u_{n}-v\right) f^{\varepsilon}-\frac{2}{\varepsilon} \int\left|v-u_{n}\right|^{2} f^{\varepsilon} \\
& -\int_{\partial \Omega \times \mathbb{R}^{d}}\left|v-u_{n}(t, x)\right|^{2} v \cdot n(x) f^{\varepsilon} \\
& \leq C_{n} \sqrt{\tilde{E}_{n}^{\varepsilon}}\left(1+\sqrt{E^{\varepsilon}}\right)-\frac{2}{\varepsilon} \tilde{E}_{n}^{\varepsilon} .
\end{aligned}
$$

This has for consequence that

$$
\left\|\tilde{E}_{n}^{\varepsilon}\right\|_{L^{1}([0, T])} \leq C_{n} \varepsilon^{2} .
$$

We eventually conclude that

$$
\tilde{E}^{\varepsilon} \longrightarrow 0 \quad \text { in } L^{1}([0, T]) .
$$




\subsection{Conclusion of the proof}

Because of (1.5), the continuity equation is true at the level $\varepsilon$

$$
\frac{\partial \rho^{\varepsilon}}{\partial t}+\operatorname{div}_{x} j^{\varepsilon}=0
$$

This equation passes to the limit to give

$$
\frac{\partial \rho}{\partial t}+\operatorname{div}_{x} j=0
$$

The Dirac structure in the limit $f$ is a consequence of the vanishing modified energy (see $(2.7))$.

Indeed for any $\phi \in C^{0}([0, T] \times \Omega), \psi \in C^{0}\left([0, T] \times \mathbb{R}^{d}\right)$, we write

$$
\begin{aligned}
\mid \int \phi(t, x) & \psi(t, v) f^{\varepsilon} d x d v d t-\int \phi \psi(t, u(t, x)) \rho^{\varepsilon} d x d t \mid \\
\leq & \int_{|v-u|<\eta}|\phi| \times|\psi(t, v)-\psi(t, u(t, x))| f^{\varepsilon} d x d v d t+\int_{|v-u| \geq \eta} \ldots \\
\leq & C\|\phi\|_{L^{\infty}([0, T] \times \Omega)} \sup _{t, v,|h|<\eta}|\psi(t, v)-\psi(t, v+h)| \times\left\|f^{\varepsilon}\right\|_{L^{1}} \\
& +\frac{2}{\eta^{2}}\|\phi\|_{L^{\infty}} \times\|\psi\|_{L^{\infty}} \int|v-u(t, x)|^{2} f^{\varepsilon} d x d v d t .
\end{aligned}
$$

Thus, the estimate (2.7) implies that

$$
\int \phi(t, x) \psi(t, v) f^{\varepsilon} d x d v d t-\int \phi \psi(t, u(t, x)) \rho^{\varepsilon} d x d t \longrightarrow 0 \quad a s \varepsilon \rightarrow 0,
$$

which is exactly point $(i)$ of Theorem 1.1. This has for immediate consequence that

$$
j^{\varepsilon} \longrightarrow j=\rho(y, x) u(t, x) .
$$

The combination of (2.10) and (2.9) gives point (ii) of Theorem 1.1.

\section{Proof of Theorem 1.2}

We first prove that System (1.14) implies Eq. (1.1) and then the converse.

\subsection{From System (1.14) to Eq. (1.1)}

Consider $\rho \in L^{\infty}\left([0, T], L^{1}(\Omega)\right)$ and $V \in L^{\infty}\left([0, T], W^{1, \infty}\left(\Omega_{t}\right)\right)$, solutions to (1.14). The derivative $\partial_{t} V$ is thus also well defined in $L^{\infty}$.

For any $R>0$ and any $\phi \in C^{1}([0, T] \times \Omega \times B(0, R))$ with $\phi(t, x, v) \geq 0, \forall x \in \partial \Omega$, $v \cdot n(x) \geq 0$, we have the following rigorous computation (thanks to the regularity 
assumptions on $\rho$ and especially $V$ )

$$
\begin{aligned}
\int\left(-\frac{\partial \phi}{\partial t}-v\right. & \left.\cdot \nabla_{x} \phi+\lambda(v-u) \cdot \nabla_{v} \phi(t, x, v)\right) \rho \delta(v-V) d x d v d t \\
= & \int\left(\left(-\frac{\partial \phi}{\partial t}-V \cdot \nabla_{x} \phi+\lambda(V-u) \cdot \nabla_{v} \phi\right)(t, x, V)\right) \rho d x d t \\
= & \int\left(-\frac{\partial(\phi(t, x, V))}{\partial t}-V \cdot \nabla_{x}(\phi(t, x, V))\right. \\
& \left.+\nabla_{v} \phi(t, x, V)\left(\frac{\partial V}{\partial t}+V \cdot \nabla_{x} V+\lambda(V-u)\right)\right) \rho d x d t \\
= & \int\left(-\frac{\partial(\phi(t, x, V))}{\partial t}-V \cdot \nabla_{x}(\phi(t, x, V))\right) \rho d x d t \\
\leq & \int \phi(0, x, V(0, x)) \rho^{0}(x) d x=\int \phi(0, x, v) \rho^{0} \delta\left(v-V^{0}(x)\right)
\end{aligned}
$$

since for all $x \in \partial \Omega$, whenever $\rho(t, x)>0, V(t, x) \cdot n(x) \geq 0$ and so $\phi(t, x, V) \geq 0$. This last inequality exactly means that $\rho \delta(v-V)$ is a solution to (1.1) in the sense of distributions. Finally, the initial condition is trivially satisfied.

\subsection{From Eq. (1.1) to System (1.14)}

We consider $\rho \in L^{\infty}\left([0, T], L^{1}(\Omega)\right)$ and $V \in L^{\infty}\left([0, T], W^{1, \infty}\left(\Omega_{t}\right)\right)$, such that $f=\rho(t, x) \delta(v-V)$ is a solution to (1.1) in the sense of distributions.

Let $R$ be large enough such that $|V(t, x)| \leq R$ for all $(t, x) \in[0, T] \times \Omega$. Let $\psi$ be any non negative function in $C_{c}^{1}\left(\mathbb{R}^{d}\right)$ with $\psi \equiv 1$ on $B(0, R)$. Then for any $\phi \in C^{1}([0, T] \times \Omega)$ with $\phi(T,)=$.0 and $\phi(t, x) \geq 0, \forall x \in \partial \Omega$, we have

$$
\begin{aligned}
\int \rho(t, x)( & \left.-\frac{\partial \phi}{\partial t}-V \cdot \nabla_{x} \phi\right) d t d x \\
= & \int \rho(t, x) \delta(v-V)\left(-\frac{\partial(\phi \psi(v))}{\partial t}-v \cdot \nabla_{x}(\phi \psi(v))\right) d x d v d t \\
= & \int \rho(t, x) \delta(v-V)\left(-\frac{\partial(\phi \psi(v))}{\partial t}-v \cdot \nabla_{x}(\phi \psi(v))\right. \\
& \left.-\lambda(u-v) \cdot \nabla_{v}(\phi \psi(v))\right) d x d v d t \\
\leq & \int \rho^{0}(x) \delta(v-V) \phi(0, x) \psi(v) d x d v=\int \rho^{0} \phi(0, x) d x,
\end{aligned}
$$

which gives the first equation and the boundary condition of (1.14). Now let us consider $\tilde{\psi} \in C_{c}^{1}\left(\mathbb{R}^{d}\right)$ with $\tilde{\psi}=v$ on $B(0, R)$. For any $\phi \in C^{1}([0, T] \times \Omega)$ with $\phi(T,)=$.0 and $\phi(t, x)=0$ for $x \in \partial \Omega$, the same kind of computation with the function $\phi \tilde{\psi}$ shows that

$$
\int \rho V(t, x)\left(\frac{\partial \phi}{\partial t}-V \cdot \nabla_{x} \phi\right) d x d t+\int \lambda(V-u) \rho \phi d x d t=\int \rho^{0} V^{0} \phi(0, x) d x .
$$

We have thus proved that $\rho$ and $V$ satisfies System (1.19) but because of the known regularity on $V$, it is equivalent to System (1.14). 


\section{Proof of Theorem $\mathbf{1 . 3}$}

We show a local existence result to (1.14) thanks to the kinetic formulation and then a priori estimate which imply the existence up to time $T^{*}$.

Let us first notice that the equation on $\rho$ in (1.14) is of course in conservative form. But if $\chi^{0}$ is a $C^{1}$ function such that $\left\{\chi^{0}(x) \neq 0\right\}=\Omega_{0}$ and if $\chi(t, x)$ is the solution to

$$
\begin{gathered}
\frac{\partial \chi}{\partial t}+V \cdot \nabla_{x} \chi=0, \\
\chi(0, x)=\chi^{0}(x),
\end{gathered}
$$

then we still have $\{\chi(t, x) \neq 0\}=\Omega_{t}$. And in particular, it is obvious that the set $\Omega_{t}$ remains open, since $\chi(t,$.$) is continuous provided V$ is regular enough.

\subsection{Local existence}

The existence time of the result, that we prove in this subsection, depends on some topological properties of the domain $\Omega_{0}$. We denote $d_{\Omega_{0}}(x, y)$ the geodesical distance on this set, that is the minimal length of the paths, completely included in the set, between two points $x$ and $y$. Since $\Omega_{0}$ has a Lipschitz boundary (assumption of Theorem 1.3), there exists a constant $\lambda_{\Omega_{0}}$ such that

$$
d_{\Omega_{0}}(x, y) \leq \lambda_{\Omega_{0}}|x-y|, \quad \forall x, y \text { in } \Omega_{0} .
$$

We now have the

Lemma 4.1 Under the assumptions of Theorem 1.3, there exist $\rho$ and $V$ solutions to (1.14), satisfying (1.17) on the time interval $\left[0, t^{*}\right]$ where $t^{*}$ depends only on $\left\|V^{0}\right\|_{W^{1, \infty}}$ and $\lambda_{\Omega_{0}}$.

\section{Proof.}

We denote $f$ the solution to (1.1) with the boundary condition (1.2) and the initial data $f^{0}=\rho^{0}(x) \delta\left(v-V^{0}(x)\right)$. Because of the regularity assumption (1.16) on $u$, the solution of this equation is expressed with the characteristics

$$
\begin{aligned}
& \partial_{t} Y(t, x, v)=W(t, x, v), \quad Y(0, x, v)=x, \\
& \partial_{t} W(t, x, v)=\lambda(u(t, Y(t, x, v))-W(t, x, v)), \quad W(0, x, v)=v .
\end{aligned}
$$

If $\Omega$ is the whole space, the system of characteristics $(Y(t, .,),. W(t, .,)$.$) is a bijection$ on $\mathbb{R}^{2 d}$. However if $\Omega$ is a bounded domain, these characteristics may leave $\Omega$ and they are not in general one-to-one on $\Omega \times \mathbb{R}^{d}$. Moreover, they are defined only if $Y(s, x, v)$ remains in $\Omega$ for all $0 \leq s \leq t$. We denote by $\mathcal{O}_{t} \subset \Omega \times \mathbb{R}^{d}$ the set of couples $(x, v)$ such that $Y(s, x, v)$ remains in $\Omega, 0 \leq s \leq t$.

The solution $f$ is given by the characteristics and vanishes at any point which they do not reach

$$
\begin{aligned}
& f(t, Y(t, x, v), W(t, x, v))=e^{d \lambda t} f^{0}(t, x, v), \quad \text { if } \quad(x, v) \in \mathcal{O}_{t}, \\
& f(t, y, w)=0, \quad \text { if } \quad \forall(x, v) \in \mathcal{O}_{t},(y, w) \neq(Y(t, x, v), W(t, x, v)) .
\end{aligned}
$$


Clearly, if $Y\left(t, ., V^{0}().\right)$ is one-to-one, i.e. if for $x_{1}, x_{2}$ in $\Omega_{0}$ with $x_{1} \neq x_{2}$, $Y\left(s, x_{1}, V^{0}\left(x_{1}\right)\right) \neq Y\left(s, x_{2}, V^{0}\left(x_{2}\right)\right)$, for $0 \leq s \leq t$, then the solution remains monokinetic on the time interval $[0, t]$.

The Lipschitz regularity of $u$, assumed in (1.16), has for consequence that $Y$ and $W$ are Lipschitz uniformly on $[0, T]$, thanks to Gronwall Lemma. We deduce that

$$
\left|\partial_{t} \nabla_{x}\left(Y\left(t, x, V^{0}(x)\right)\right)\right| \leq C\left(1+\left\|\nabla V^{0}\right\|_{L^{\infty}}\right), \quad \forall x \in \Omega_{0},
$$

where $C$ depends only on the norm of $u$ in $L^{\infty}\left([0, T], W^{1, \infty}\left(\mathbb{R}^{d}\right)\right)$. Therefore

$$
\left|Y\left(t, x_{1}, V^{0}\left(x_{1}\right)\right)-Y\left(t, x_{2}, V^{0}\left(x_{2}\right)\right)\right| \geq\left|x_{1}-x_{2}\right|\left(1-C t \lambda_{\Omega_{0}}\left(1+\left\|V^{0}\right\|_{W^{1, \infty}}\right)\right),
$$

and so if

$$
t^{*} \leq \frac{C}{\lambda_{\Omega_{0}}\left(1+\left\|V^{0}\right\|_{W^{1, \infty}}\right)},
$$

the solution $f$ remains monokinetic on $\left[0, t^{*}\right]$, i.e. equal to $\rho(t, x) \delta(v-V(t, x))$.

Since the mapping $Y\left(t, ., V^{0}().\right)$ is Lipschitz on $\Omega_{0}$ and since $\Omega_{0}$ is regular enough, we may extend it on all $\mathbb{R}^{d}$ by a Lipschitz mapping which we still denote $Y\left(t, ., V^{0}().\right)$. This extension is such that if $x \notin \Omega$ then for all $t>0, Y\left(t, x, V^{0}(x)\right) \notin \Omega$. The set $\Omega_{t}$ is exactly the intersection of the image of the initial set $\Omega_{0}$ by the mapping $Y(t,$. , $\left.V^{0}().\right)$, and of $\Omega$. In particular, because of the regularity of the characteristics, this set $\Omega_{t}$ is an open subdomain of $\Omega$, with a Lipschitz boundary, indeed the boundary of $\Omega_{t}$ is made of pieces of the boundary of the image of $\Omega_{0}$ linked with pieces of $\partial \Omega$. So, we remark here that in general $\Omega_{t}$ cannot be more than Lipschitz regular.

The function $V(t, x)$ is equal to $W\left(t, x, V^{0}(x)\right) \circ\left(Y\left(t, ., V^{0}(.)\right)\right)^{-1}$ and thus this function belongs to $L^{\infty}\left([0, T], W^{1, \infty}\left(\Omega_{t}\right)\right)$.

Eventually, since $\rho=\int f d x$, it trivially belongs to $L^{\infty}\left([0, T], M^{1}(\Omega)\right)$. But since $\rho$ satisfies the first equation of System (1.14) and thanks to the regularity of $V$, any integral $\int \beta(\rho) d x$ (for a regular and non-negative function $\beta$ ) remains bounded if it was so initially. Consequently $\rho$ belongs in fact to $L^{\infty}\left(\left[0, t^{*}\right], L^{1}(\Omega)\right)$ and the lemma is proved.

\section{$4.2 \quad$ A priori estimates}

To obtain existence globally in time, we only have to prove that $\|V(t, .)\|_{W^{1, \infty}}$ and the ratio of the geodesical distance, over the euclidian one, are bounded, a priori, on any time interval.

We benefit here from the local aspect of System (1.14). It is indeed possible to show that the field $V$ is Lipschitz uniformly in time, independently of the exact form of the set $\Omega_{t}$ and of this ratio of distance (provided the set remains regular enough, i.e. Lipschitz and so before the first folding time).

Lemma 4.2 Assume that

$$
\left\|\nabla V^{0}\right\|_{L^{\infty}\left(\Omega_{0}\right)} \leq \frac{\lambda}{2 d}, \quad\|u\|_{L^{\infty}\left([0, T], W^{1, \infty}(\Omega)\right)} \leq \frac{\lambda}{4 d} .
$$


Then at any time $t$, as long as $\Omega_{s}$ was Lipschitz on $0 \leq t \leq s$

$$
\|V(t, .)\|_{W^{1, \infty}\left(\Omega_{t}\right)} \leq\left\|V^{0}\right\|_{W^{1, \infty}\left(\Omega_{0}\right)}+2\|\nabla u\|_{L^{\infty}([0, T] \times \Omega)} .
$$

\section{Proof.}

The first point to note is that, because of the evolution equation on $V$ in System (1.14), we trivially know that

$$
\|V(t, .)\|_{L^{\infty}\left(\Omega_{t}\right)} \leq\left\|V^{0}\right\|_{L^{\infty}\left(\Omega_{0}\right)}+\|u\|_{L^{\infty}([0, T] \times \Omega)} .
$$

At any point $x \in \Omega_{t}$, since $\Omega_{t}$ is open, we have

$$
\frac{\partial\left(\partial_{i} V\right)}{\partial t}+V \cdot \nabla_{x} \partial_{i} V=-\partial_{i} V \cdot \nabla_{x} V+\lambda\left(\partial_{i} u-\partial_{i} V\right) .
$$

We define the flow $Y(t, x)$ by

$$
\frac{\partial Y}{\partial t}=V(t, Y(t, x)), \quad Y(0, x)=0 .
$$

The flow is well defined for any $x \in \bar{\Omega}_{0}=\left\{x \in \Omega_{0} \mid Y(s, x) \in \Omega, 0 \leq s \leq t\right\}$ and $\Omega_{t}$ is exactly the intersection of the image of $\Omega_{0}$ by this flow and of $\Omega$. Let us denote

$$
W_{i j}(t, x)=\partial_{i} V_{j}(t, Y(t, x)), \quad U_{i j}(t, x)=\partial_{i} u_{j}(t, Y(t, x)) .
$$

We have

$$
\frac{\partial W}{\partial t}=-W \times W+\lambda(U-W)
$$

Let $i_{0}, j_{0}$ be such that at time $t$

$$
\left|W_{i_{0} j_{0}}(t)\right|=\sup _{i, j}\left|W_{i, j}(t)\right| .
$$

Let us assume that $W_{i_{0} j_{0}}$ is positive (the negative case yields the same result). We deduce the bound

$$
\begin{aligned}
\frac{\partial W_{i_{0} j_{0}}}{\partial t} & \leq d W_{i_{0} j_{0}}^{2}+\lambda\left(U_{i_{0} j_{0}}-W_{i_{0} j_{0}}\right) \\
& \leq W_{i_{0} j_{0}}\left(d W_{i_{0} j_{0}}-\frac{\lambda}{2}\right)+\lambda\left(U_{i_{0} j_{0}}-\frac{1}{2} W_{i_{0} j_{0}}\right) .
\end{aligned}
$$

We may deduce from this that if $W_{i_{0} j_{0}}$ is smaller than $\lambda / 2 d$ and larger than $2 U_{i_{0} j_{0}}$, then $\partial_{t} W_{i_{0} j_{0}}$ is negative. Because of the hypothesis of the lemma and of the equivalent result if $W_{i_{0} j_{0}}<0$, this implies that

$$
\sup _{i, j}\left|W_{i j}(t)\right| \leq 2 \sup _{i, j, t}\left|U_{i j}(t)\right|+\sup _{i, j}\left|W_{i j}(0)\right| .
$$

Recalling that the flow $Y$ is a bijection from $\bar{\Omega}_{0}$ to $\Omega_{t}$, this proves the lemma.

This lemma clearly shows that all the trouble comes from $\Omega_{t}$ and more precisely $\partial \Omega_{t}$ because locally inside $\Omega_{t}$ the equations are always well defined. The combination of the lemma with the short time result 4.1 proves the existence up to time $T^{*}$. 


\section{Proof of Theorem 1.4}

We begin with the proof of the point (ii). A first remark is that Lemma 4.2 implies that there exists a constant $C$ depending only on $V^{0}$ and $u$ such that, for any $\varepsilon$ large enough and any time $t$

$$
\left\|V_{\varepsilon}(t, .)\right\|_{W^{1, \infty}\left(\Omega_{t}^{\varepsilon}\right)} \leq C .
$$

For any $\phi^{0} \in W^{1, \infty}(\Omega)$, nonnegative whose support is included in $\Omega_{0}$, the solution $\phi_{\varepsilon}$ to

$$
\begin{aligned}
& \partial_{t} \phi_{\varepsilon}+V_{\varepsilon} \cdot \nabla_{x} \phi_{\varepsilon}=0, \\
& \phi_{\varepsilon}(t=0, x)=\phi^{0},
\end{aligned}
$$

is bounded in $W^{1, \infty}([0, T] \times \Omega)$ independently of $\varepsilon$ (but the bound depends on $\phi^{0}$ ) and its support is included in $\Omega_{t}^{\varepsilon}$. We compute for any $(t, x) \in[0, T] \times \Omega$

$$
\begin{aligned}
\frac{d}{d t} \phi_{\varepsilon}\left|V_{\varepsilon}-u\right|(t, x)= & -\left|V_{\varepsilon}-u\right|^{2} V_{\varepsilon} \cdot \nabla_{x} \phi_{\varepsilon}-2 \phi \frac{V_{\varepsilon}-u}{\left|V_{\varepsilon}-u\right|} \cdot \partial_{t} u \\
& -2 \phi\left(V_{\varepsilon} \cdot \nabla_{x}\right) V_{\varepsilon} \cdot \frac{V_{\varepsilon}-u}{\left|V_{\varepsilon}-u\right|}-\frac{2}{\varepsilon} \phi\left|V_{\varepsilon}-u\right| \\
\leq & C-\frac{2}{\varepsilon} \phi\left|V_{\varepsilon}-u\right|(t, x),
\end{aligned}
$$

where $C$ is again a constant independent of $\varepsilon$. This implies that, given any $t^{*}>0$, there exist $C_{t^{*}}$ such that

$$
\left\|\phi_{\varepsilon}\left|V_{\varepsilon}-u\right|\right\|_{L^{\infty}\left(\left[t^{*}, T\right] \times \Omega\right)} \leq C_{t^{*}} \varepsilon .
$$

Choosing a sequence $\phi_{n}^{0}$ converging toward $\mathbf{I}_{\Omega_{0}}$ in every $L^{p}(\Omega), p<\infty$, we obtain a sequence $\phi_{\varepsilon, n}$ converging toward $\mathbf{I}_{\Omega_{t}^{\varepsilon}}$ and we deduce the point (ii).

The first point $(i)$ is now only a trivial consequence. Since

$$
\left\|\rho_{\varepsilon}\right\|_{L^{\infty}\left([0, T], L^{1}(\Omega)\right)} \leq\left\|\rho^{0}\right\|_{L^{1}(\Omega)},
$$

we may extract a subsequence converging weakly toward $\rho$ in $M^{1}(\Omega)$. By a suitable regularization of $\rho_{\varepsilon}$, we may show that $\rho_{\varepsilon} V_{\varepsilon}$ converges toward $\rho u$ weakly in $M^{1}(\Omega)$, just like we have just done for $\mathbf{I}_{\Omega_{t}^{\varepsilon}} V_{\varepsilon}$.

As for the point $(i i i)$, for any $\phi_{\varepsilon} \in W^{1, \infty}([0, T] \times \Omega)$ whose support in $x$ is always included in $\Omega_{t}^{\varepsilon}$, because of the second equation in System (1.21)

$$
\begin{aligned}
\phi_{\varepsilon}\left(\frac{V_{\varepsilon}-u}{\varepsilon}+\partial_{t} u+u \cdot \nabla_{x} u\right)= & \phi_{\varepsilon} \partial_{t}\left(u-V_{\varepsilon}\right)+\phi_{\varepsilon}\left(u-V_{\varepsilon}\right) \cdot \nabla_{x} u \\
& +\phi_{\varepsilon} V_{\varepsilon} \cdot \nabla_{x}\left(u-V_{\varepsilon}\right) \\
= & \partial_{t}\left(\phi_{\varepsilon}\left(u-V_{\varepsilon}\right)\right)-\mathbf{I}_{\Omega_{t}^{\varepsilon}}\left(u-V_{\varepsilon}\right) \partial_{t} \phi_{\varepsilon} \\
& +\phi_{\varepsilon}\left(u-V_{\varepsilon}\right) \cdot \nabla_{x} u+\operatorname{div}_{x}\left(\phi_{\varepsilon} V_{\varepsilon} \otimes\left(u-V_{\varepsilon}\right)\right) \\
& -\mathbf{I}_{\Omega_{t}^{\varepsilon}}\left(u-V_{\varepsilon}\right) \operatorname{div}_{x}\left(\phi_{\varepsilon} V_{\varepsilon}\right) .
\end{aligned}
$$


From the point ( $i i)$ and the fact that $\phi_{\varepsilon}$ is bounded in $W^{1, \infty}$ uniformly in $\varepsilon$ as $\nabla_{x} V$ is in $L^{\infty}$, the point (iii) follows easily.

\section{References}

[1] F. Bouchut and F. James, Duality solutions for pressureless gases, monotone scalar conservation laws, and uniqueness. Comm. Partial Differential Equations, 24 (1999), 2173-2189.

[2] Y. Brenier, A Vlasov-Poisson type formulation of the Euler equations. INRIA reports 1070 (1989).

[3] Y. Brenier, Convergence of the Vlasov-Poisson system to the incompressible Euler equations. Comm. Partial Differential Equations, 25 (2000), 737-754.

[4] C. Dietz and V. Sandor, The hydrodynamical limit of the Vlasov-Poisson system. Transport Theory Statist. Phys. , 28 (1999), 499-520.

[5] E. Frénod and E. Sonnendrücker, Long time behavior of the two-dimensional Vlasov equation with a strong external magnetic field. Math. Models Methods Appl. Sci., 10 (2000), 539-553.

[6] F. Golse and L. Saint-Raymond, The Vlasov-Poisson system with strong magnetic field. J. Math. Pures Appl., 9 (1999), 791-817.

[7] T. Goudon, Asymptotic problems for a kinetic model of two-phase flow. To appear in Proc. Roy. Soc. Edinburgh.

[8] W. Greenberg, C. Van der Mee and V. Protopopescu, Boundary value problems in abstract kinetic theory. Birkhäuser Verlag 1987.

[9] E. Grenier, Defect measures of the Vlasov-Poisson system. Comm. Partial Differential Equations, 21 (1996), 363-394.

[10] E. Grenier, Limite quasineutre en dimension 1. Journées "Équations aux Dérivées Partielles" (Saint-Jean-de-Monts 1999), Exp II, Univ. Nantes, 1999.

[11] H. Herrero, B. Lucquin-Desreux and B. Perthame, On the motion of dispersed bubbles in a potential flow- a kinetic description of the added mass effect. SIAM J. Appl. Math., 60 (2000), 61-83.

[12] P.E. Jabin, Macroscopic limit of Vlasov type equations with friction. Ann. Inst. H. Poincaré Anal. Non Linéaire, 17 (2000), 651-672.

[13] P.E. Jabin and B. Perthame, Notes on mathematical problems on the dynamics of dispersed particles interacting through a fluid. Modeling in applied sciences, 111-147, Model. Simul. Sci. Eng. Technol., Birkhäuser Boston, 2000. 
[14] P.L. Lions, Mathematical topics in fluid mechanics, vol. 1 incompressible models. Oxford Lecture Series in Mathematics and its Applications, Oxford University Press 1996.

[15] A. Majda and Y. Zheng, Existence of global weak solutions to one-component Vlasov-Poisson and Fokker-Planck-Poisson systems in one space dimension with measures as initial data. Comm. Pure Appl. Math., 47 (1994), 1365-1401.

[16] O. Runborg, Some new results in multiphase geometrical optics. M2AN Math. Model. Numer. Anal., 34 (2000), 1203-1231.

[17] P. Smereka, A Vlasov description of the Euler equation. Nonlinearity, 9 (1996), 1362-1386.

[18] D.B. Taulbee and C.P. Yu, A theory of aerosol deposition in the human respiratory tract. J. Appl. Physiol., 38 (1975), 77-85. 